\title{
NOUVELLE
}

\section{Le récepteur NTS2 : un frein à la douleur}

Philippe Sarret, Nicolas Beaudet, Geneviève Roussy
Département de physiologie et biophysique, Faculté de médecine, Université de Sherbrooke, 3001, $12^{\mathrm{e}}$ Avenue Nord, Sherbrooke (Québec), JIH 5N4 Canada.

Philippe.sarret@usherbrooke.ca
$>$ Qu'est-ce que la douleur? II est souvent très difficile de définir la douleur en raison de sa nature très subjective. Pensons, par exemple, à la dernière douleur que nous avons endurée. À quoi était-elle due? Syndrome abdominal aigu, maux de dos, migraines, douleur postopératoire ou post-traumatique, douleur chronique récurrente sans diagnostic bien précis? Que peut-on dire de son intensité et de son acuité ? Quelle était la sensation douloureuse ressentie? Pouvait-on parler de brûlure, de picotement, de décharge électrique ou encore de crampes? À l'évidence, si nous sommes tous un jour confrontés à la douleur, nous ne la ressentons pas et n'en parlons pas de la même façon. L'Association internationale pour l'étude de la douleur définit ainsi la douleur: «une expérience sensorielle et émotionnelle désagréable, associée ou non à des lésions réelles ou potentielles, ou décrite en des termes évoquant de tels dommages ».

La douleur est généralement répertoriée comme aiguë ou chronique. La douleur aiguë est considérée comme un signal d'alarme pour l'organisme afin qu'il déclenche des réactions de prévention et de protection rapides. Si la douleur aiguë a pour finalité biologique la préservation de l'intégrité de l'organisme, il n'y a aucun intérêt physiologique dans la douleur chronique. Les stimulus algiques aigus intenses peuvent en peu de temps provoquer des altérations fonctionnelles et structurelles rémanentes, modifiant à long terme la transmission et l'élaboration des signaux nociceptifs. Les douleurs aiguës non adéquatement traitées peuvent évoluer vers la chronicisation

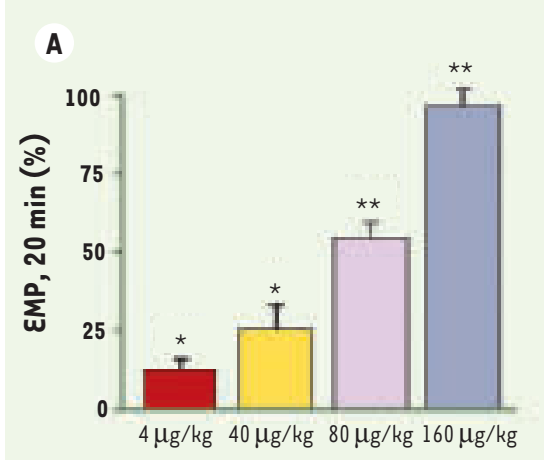

B

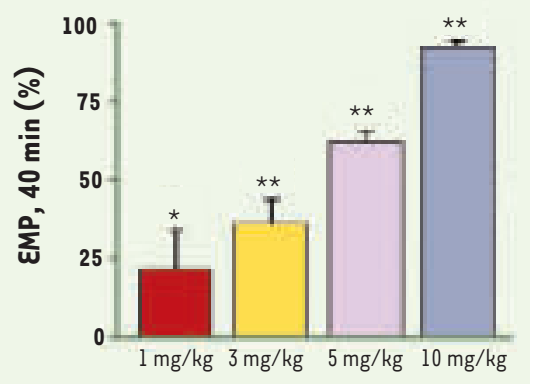

Figure 1. Comparaison des propriétés antinociceptives d'un agoniste du récepteur NTS2 et de la morphine dans un test de douleur aiguë. Le stimulus thermique est provoqué par l'immersion de la queue du rat dans de l'eau à $52^{\circ} \mathrm{C}$ (tail-flick test). A. Effet de l'injection intrathécale de l'agoniste du récepteur NTS2, le JMV-431, sur la réponse analgésique [6]. B. Administration par voie intrapéritonéale de la morphine [10]. L'effet maximal possible (EMP), caractérisé par une augmentation du temps de réponse de l'animal, observé avec le JMV-431, est équivalent à celui obtenu avec la morphine. Les différences significatives sont indiquées par des astérisques: ${ }^{*}<$ 0,$05 ; * \star p<0,001$.

par altérations neuroplastiques. Dans un tel contexte, la douleur a perdu toute valeur protectrice. Elle est destructrice tant sur le plan physique que psychologique et social. Aiguë, persistante et chronique, la douleur s'étage donc selon un continuum subtil tant sur le plan physiopathologique que clinique: la transition entre différents états ne doit pas être négligée. Dans bien des circonstances, un traitement médicamenteux précoce, continu et à doses appropriées peut contribuer à prévenir l'évolution vers un syndrome douloureux chronique [1].

Le choix de l'antalgique ou de la substance anti-douleur dépend de ses caractéristiques pharmacocinétiques, et, bien sûr, de la nature et de l'intensité de la douleur à soulager. Les opioïdes tels que la morphine ou le fentanyl sont utilisés comme analgésiques depuis des siècles et leurs associations aux anti-inflammatoires non-stéroïdiens, anxiolytiques, antidépresseurs ou encore antiépileptiques constituent actuellement le traitement de choix pour soulager les douleurs modérées à sévères. Cependant, il n'en reste pas moins que de nombreuses formes de douleur ne sont pas atténuées par les antalgiques communs. De plus, les patients qui répondent au traitement sont susceptibles de l'interrompre en raison des effets secondaires intolérables de ces médicaments: nausées, constipation, dépression respiratoire ou, à plus long terme, dépendance et tolérance.

Parmi les neurotransmetteurs endogènes qui interviennent dans la modulation des messages nociceptifs, on trouve la neurotensine (NT), un neuropeptide de 13 acides aminés, découvert sur la base de ses propriétés vasodi- 
latatrices [2]. De nombreuses études révèlent, en effet, que l'administration centrale ou spinale de la NT se traduit par une réponse analgésique, insensible aux antagonistes opiö̈dergiques comme la naloxone [3]. Parmi les trois récepteurs de la NT (NTSl-3) connus à ce jour $[4,5]$, nous avons récemment démontré que le récepteur NTS2 jouait un rôle important dans la réponse antinociceptive déclenchée au niveau spinal [6]. Chez le rat, l'administration intrathécale d'agonistes sélectifs du récepteur NTS2 diminue de façon considérable la sensibilité provoquée par un stimulus thermique nociceptif (Figure 1). Parallèlement, l'inactivation du récepteur NTS2 par injection intracérébroventriculaire d'oligonucléotides antisens réduit les effets analgésiques de la NT en réponse aux stimulus nociceptifs déclenchés par l'injection abdominale d'acide acétique [7]. La présence du récepteur
NTS2 à des endroits stratégiques pour effectuer un contrôle central et spinal des voies de la douleur corrobore ces effets physiologiques. Le récepteur NTS2 est localisé en particulier dans les régions où s'effectuent la transmission et la modulation du message nociceptif, telles que les ganglions rachidiens, la corne dorsale de la moelle épinière, la substance grise périaqueducale, la région bulbaire rostro-ventrale et le noyau réticulé paragigantocellulaire [6, 8] (Figure 2).

L'émotion comme l'attention peuvent avoir un effet pro- ou antinociceptif selon le contexte. Ces modulations font intervenir plusieurs zones corticales en particulier limbiques (amygdale), et des zones sous corticales comme le thalamus. La présence du récepteur NTS2 dans ces structures cérébrales laisse penser qu'il pourrait aussi participer à l'aspect émotionnel de la réponse nociceptive, lequel affecte
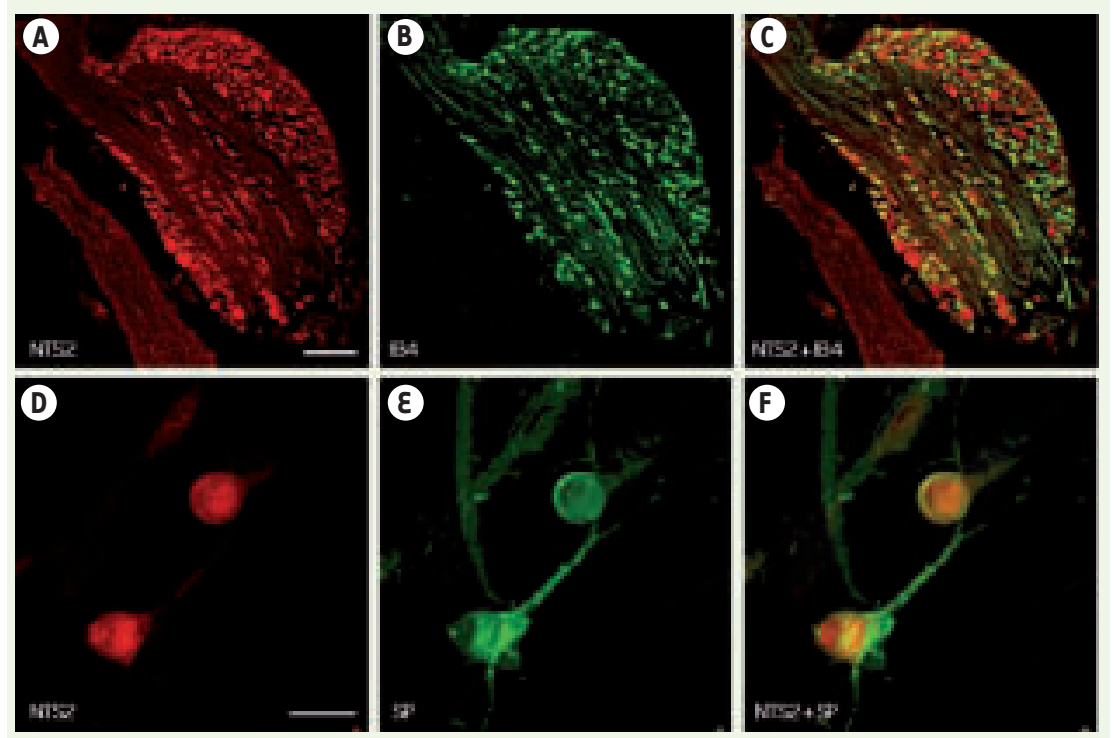

Figure 2. Distribution du récepteur NTS2 dans les ganglions spinaux. A-C. Distribution immunohistochimique du récepteur NTS2 dans les corps cellulaires des neurones des ganglions des racines dorsales de la moelle épinière. La co-localisation du récepteur NTS2 avec le marqueur isolectine B4 (IB4) révèle que celui-ci est présent dans les neurones périphériques non peptidergiques qui forment les fibres nerveuses amyéliniques de type C. D-F. Culture de neurones de ganglions rachidiens. La détection du récepteur NTS2 dans des neurones sensoriels immunopositifs pour la substance $P$ indique que le récepteur NTS2 est également exprimé dans les neurones peptidergiques des fibres de type C. La présence du récepteur NTS2 dans les ganglions rachidiens vient confirmer sa participation à la transmission des messages nociceptifs.

fortement la douleur chronique. Par ailleurs, le récepteur NTS2 pourrait également jouer un rôle important dans la physiopathologie des douleurs chroniques d'origine neuropathique. L'étude récente du groupe de Zhang et al. [9] est particulièrement prometteuse à cet égard. En effet, ces chercheurs ont démontré que le profil d'expression du récepteur NTS2 dans les ganglions rachidiens de la région lombaire de la moelle épinière était affecté chez des rats dont le nerf sciatique avait été sectionné. Même si les mécanismes par lesquels les récepteurs NTS2 modulent les impulsions nociceptives ne sont encore que spéculatifs, il ne fait cependant aucun doute que les agonistes des récepteurs de la NT pourraient représenter une nouvelle classe d'analgésiques efficaces pour le traitement de la douleur aiguë et chronique. $\diamond$

NTS2 receptor:

slamming the brakes on pain

\section{RÉFÉRENCES}

1. Beaulieu P. Pharmacologie de la douleur. Montréal: Presses de l'Université de Montréal, 2005.

2. Rostene WH, Alexander MJ. Neurotensin and neuroendocrine regulation. Front Neuroendocrinol $1997 ; 18: 115-73$.

3. Dobner PR. Multitasking with neurotensin in the central nervous system. Cell Mol Life Sci 2005 ; 62 : 1946-63.

4. Sarret $P$, Beaudet A. Neurotensin receptors in the central nervous system. In: Handbook of chemical neuroanatomy, vol. 20. Peptide receptors, part II. Amsterdam : Elsevier, 2003 : 323-400.

5. Vincent JP, Mazella J, Kitabgi P. Neurotensin and neurotensin receptors. Trends Pharmacol Sci 1999 ; $20: 302-9$

6. Sarret $P$, Esdaile MJ, Perron $A$, et al. Potent spinal analgesia elicited through stimulation of NTS2 neurotensin receptors. J Neurosci 2005 ; 25 : 8188-96.

7. Dubuc I, Sarret P, Labbe-Jullie C, et al. Identification of the receptor subtype involved in the analgesic effect of neurotensin.J Neurosci 1999; 19 : 503-10.

8. Sarret P, Perron A, Stroh T, Beaudet A. Immunohistochemical distribution of NTS2 neurotensin receptors in the rat central nervous system. J Comp Neurol 2003 ; 461 : 520-38.

9. Yang L, Zhang FX, Huang F, et al. Peripheral nerve injury induces trans-synaptic modification of channels, receptors and signal pathways in rat dorsal spinal cord. Eur J Neurosci 2004 ; 19 : 871-83.

10. Morinville A, Cahill CM, Esdaile MJ, et al. Regulation of delta-opioid receptor trafficking via mu-opioid receptor stimulation : evidence from mu-opioid receptor knock-out mice. J Neurosci 2003 ; 23 : 4888-98. 